\title{
Effects of Heat and Moisture Exchangers and Exhaled Humidity on Aerosol Deposition in a Simulated Ventilator-Dependent Adult Lung Model
}

\author{
Arzu Ari PhD RRT PT CPFT FAARC, Khalid S Alwadeai MSc RRT-NPS, and \\ James B Fink PhD RRT FAARC
}

\begin{abstract}
BACKGROUND: Many in vitro models report higher inhaled dose with dry versus heated humidity. Heat-and-moisture exchangers (HMEs) provide passive humidity in ventilator-dependent patients but act as a barrier to aerosol. The HMEs designed to allow aerosol delivery (HME-ADs) have not been well described. The purpose of this study is to determine the impact on aerosol deposition of HME-ADs with and without active exhaled humidity in a simulated ventilator-dependent adult model. METHODS: We used an in vitro lung model consisting of an intubated teaching mannequin with an endotracheal tube of $8.0 \mathrm{~mm}$ inner diameter with bronchi directly attached to a collecting filter and passive rubber test lung to provide testing without active exhaled humidity. To simulate exhaled humidity, a Cascade humidifier $\left(37^{\circ} \mathrm{C}\right.$ and $100 \%$ relative humidity) was placed between the collecting filter and test lung, simulating body temperature and pressure saturated exhaled humidity at the bronchi. Albuterol sulfate $(2.5 \mathrm{mg} / 3 \mathrm{~mL}$ ) was administered with a mesh nebulizer (Aerogen Solo) placed in the inspiratory limb of the ventilator circuit at the Y-piece, with no HME in place (control) and with 3 HME-AD devices, including the CircuVent, Humid-Flo, and AirLife, with and without exhaled humidity. Drug was eluted from the collecting filter and analyzed with spectrophotometry. Student $t$ tests and analysis of variance were used for data analysis $(P<.05)$. RESULTS: The percentage of drug dose delivered (mean \pm SD) distal to the bronchi in the control experiments was greater than all of the HME-ADs without exhaled humidity $18 \pm 0.7$ and with active exhaled humidity $10.8 \pm 0.2 \%(P<.005)$. Without exhaled humidity, aerosol delivery with the CircuVent $(12.6 \pm 0.8)$, Humid-Flo $(15.3 \pm 0.8)$, and AirLife $(12.0 \pm 0.5)$ was less than control $(P<.001, P=.01$ and $P<.001$, respectively). In contrast, with exhaled humidity, no difference was found between control and HME-ADs $(P=.89)$. Also, a greater variation between control and the 3 HME-ADs was observed without exhaled humidity. Drug delivery without exhaled humidity exceeded aerosol deposition obtained with exhaled humidity in all conditions tested in this study. CONCLUSIONS: In this model simulating active exhaled humidity, aerosol drug delivery was lower and more consistent with both control and the HME-ADs than with the standard nonhumidified model. Further studies are needed to determine whether greater deposition in a dry model is an artifact of the model that does not simulate exhaled humidity. Key words: Heat-and-moisture exchanger; humidification; nebulizers; aerosols; mechanical ventilation; inhalation therapy. [Respir Care 2017;62(5):538-543. (C) 2017 Daedalus Enterprises]
\end{abstract}

Introduction

Providing heated and humidified gas during mechanical ventilation is important for the pulmonary function of ventilator-dependent patients because their upper airway is bypassed during intubation. The consequences of poor humidification include hypothermia, disruption of the airway epithelium, bronchospasm, atelectasis, airway obstruction, and occlusion of the endotracheal tube due to inspissation of airway secretions. ${ }^{1}$ To prevent poor humidification during mechanical ventilation, heat and humidification should be provided at body temperature and pressure saturated with water vapor with $37^{\circ} \mathrm{C}$ and $100 \%$ relative humidity. The International Organization for Standardization stated 


\section{Effects of HME on Aerosol Deposition in Simulated Ventilation}

a potential thermal hazard to the patient with the use of gas temperature $>37^{\circ} \mathrm{C} .{ }^{2,3}$ Inspiring gas with a temperature $>37^{\circ} \mathrm{C}$ and a relative humidity at $100 \%$ results in condensation, low viscosity mucus, and excessive peripheral fluid that may lead to a reduction in mucociliary transport velocity. ${ }^{1}$

Airway humidification can be accomplished via a heated humidifier or heat-and-moisture exchangers (HMEs), also known as artificial noses, that are placed between the endotracheal tube (ETT) and the Y-piece in the ventilator circuit. They collect heat and moisture from the patient's breath during expiration and use it to warm and humidify the subsequent inspired breath. Therefore, HMEs are considered to be passive humidifiers as opposed to heated humidifiers, which are also known as active humidifiers. ${ }^{1}$

There are several advantages of HMEs over heated humidifiers. For instance, HMEs are portable, simple to use, and cost-effective to maintain during mechanical ventilation because they do not require electrical power and large volumes of water as do heated humidifiers. Previous research has reported that using the HME in the ventilator circuit up to $7 \mathrm{~d}$ may be safe and does not increase the risk of ventilator-associated pneumonia. ${ }^{4,5}$

HMEs also have some disadvantages. For example, use of an HME in the ventilator circuit increases air-flow resistance during mechanical ventilation, although the clinical importance of an increase in air-flow resistance is often negligible. ${ }^{6}$ Delivering aerosolized medications through HMEs during mechanical ventilation has been problematic because they can drastically decrease aerosol deposition to the airway and increase airway resistance in ventilator-dependent patients. Most manufacturing companies suggest removing HMEs from the ventilator circuit before aerosol therapy, resulting in patients being disconnected from the ventilator during the removal and reinsertion of the HME from the ventilator circuit, interrupting mean airway pressures and PEEP. These interruptions of pressure are associated with lung de-recruitment that may require $1 \mathrm{~h}$ or more to return the lung to previous volumes. $^{7,8}$ Also, disconnecting the patient from the ventila-

The authors are affiliated with the Department of Respiratory Therapy, Georgia State University, Atlanta, Georgia 30302-4019.

Dr Ari has disclosed relationships with Bayer Pharmaceuticals and ARC Medical. Dr. Fink has disclosed relationships with Aerogen, Dance Biopharm, Parion, Bayer, and Ansun. Mr Alwadeai has disclosed no conflicts of interest.

Correspondence: Arzu Ari PhD RRT PT CPFT FAARC, Department of Respiratory Therapy, Georgia State University, P.O. Box 4019, Atlanta, GA 30302-4019. E-mail: arzuari@ hotmail.com.

DOI: $10.4187 /$ respcare. 05015

\section{QUICK LOOK}

\section{Current knowledge}

Heat-and-moisture exchangers (HMEs) placed between the endotracheal tube and the Y-piece in the ventilator circuit can filter and reduce medical aerosol delivery. Removing HMEs from the ventilator circuit before aerosol therapy causes lung de-recruitment and increases the risk of infection for both patient and care providers. HMEs designed for use with aerosol delivery (HMEADs) have 2 configurations: (1) the aerosol configuration and (2) the HME configuration. In the aerosol configuration, the inspiratory gas is directed to bypass the HME portion of the device.

\section{What this paper contributes to our knowledge}

Aerosol drug delivery with a model simulating exhaled heat and humidity showed no difference between control (no HME) and all 3 of the HME-ADs. Our findings suggest that the model without active exhaled heated humidity probably overestimates aerosol delivery distal to the airways compared with active exhaled humidity.

tor may increase the risk of infection for both patient and care providers. ${ }^{9,10}$

Specialty HMEs designed to accommodate aerosol delivery (HME-ADs) have been designed and manufactured to alleviate issues in aerosol drug delivery associated with regular HMEs. The new HME-ADs have 2 configurations: (1) the aerosol configuration and (2) the HME configuration. Whereas the HME configuration works like a regular HME, in the aerosol configuration, the inspiratory gas is directed in such a way as to bypass the HME portion of the device.

Since the introduction of HME-ADs, no information has been available regarding their impact on efficiency of aerosol delivery during mechanical ventilation. Because HMEs rely on recovering heat and water from exhaled air to function, we modified a standard passive test lung to simulate heat and humidity exhaled in ventilated patients. We hypothesized that HME-ADs would reduce aerosol delivery compared with control and that measured aerosol delivery would be lower with exhaled humidity. The purpose of this study was to determine the effect of HME-ADs with and without active exhaled humidity on aerosol deposition in a simulated ventilator-dependent adult model.

\section{Methods}

\section{Lung Model}

An in vitro lung model was used, consisting of an intubated teaching mannequin with an endotracheal tube of 

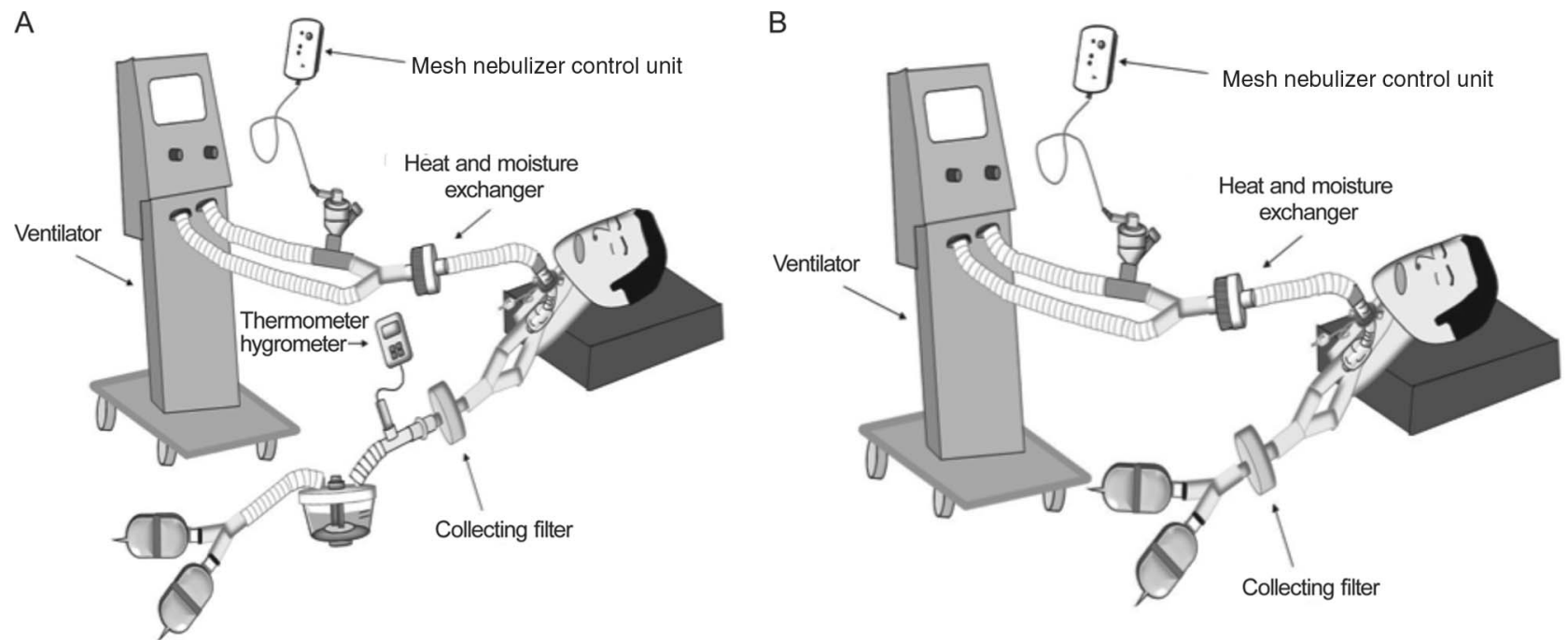

Fig. 1. Experimental set-up of the study with $(A)$ and without $(B)$ exhaled humidity. The heat and moisture exchanger was designed for aerosol delivery.

$8.0 \mathrm{~mm}$ inner diameter with bronchi directly attached to a collecting filter (Respirgard II, Vital Signs, Totowa, New Jersey) and rubber test lung to provide testing without active exhaled humidity (Fig. 1). To simulate exhaled humidity, the collecting filter was connected to a Cascade humidifier (Covidien-Puritan Bennett, Boulder, Colorado) set to deliver $37 \pm 2{ }^{\circ} \mathrm{C}$ and $95-100 \%$ relative humidity, simulating body temperature and pressure saturated exhaled humidity at the bronchi, as verified with a digital hygrometer/thermometer (Control Company, Friendswood, Texas). The position of the collecting filter was superior to the ETT to minimize condensate reaching the filter medium.

\section{Data Collection}

Albuterol sulfate $(2.5 \mathrm{mg} / 3 \mathrm{~mL})$ was administered with a mesh nebulizer (Aerogen Solo, Aerogen Ltd, Galway, Ireland) placed in the inspiratory limb at the Y-piece, with no HME in place (control) and with 3 HME-AD devices (Fig. 2), including the CircuVent (Smiths-Medical, Keene, New Hampshire), Humid-Flo (Hudson-RCI, Arlington Heights, Illinois), and AirLife (CareFusion, San Diego, California), with and without exhaled humidity. Table 1 includes the main characteristics of studied HME-ADs, including weight, dead space, and moisture output.

Before testing each HME-AD, it was placed between ETT and the Y-piece in the circuit using the HME configuration, and airway resistance was measured every $5 \mathrm{~min}$ until the resistance stabilized and no further change occurred (Table 2). All of the HME-ADs had not further increase in resistance after $10 \mathrm{~min}$ in the ventilator circuit.

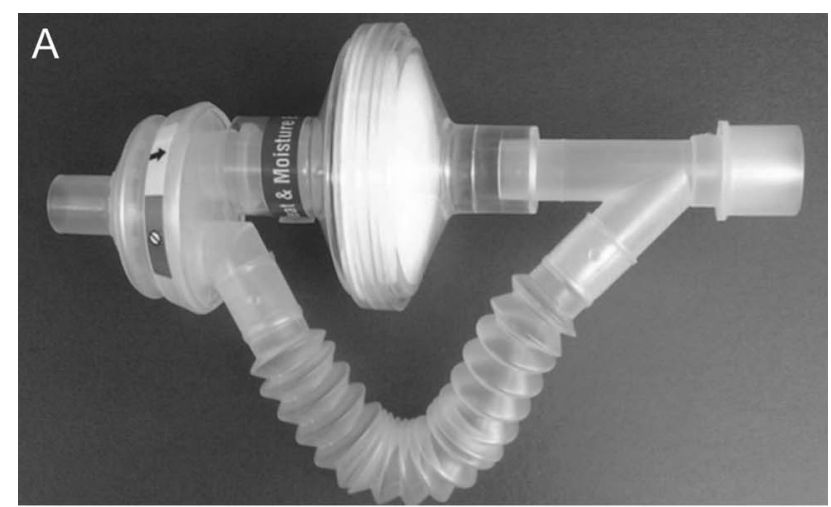

B
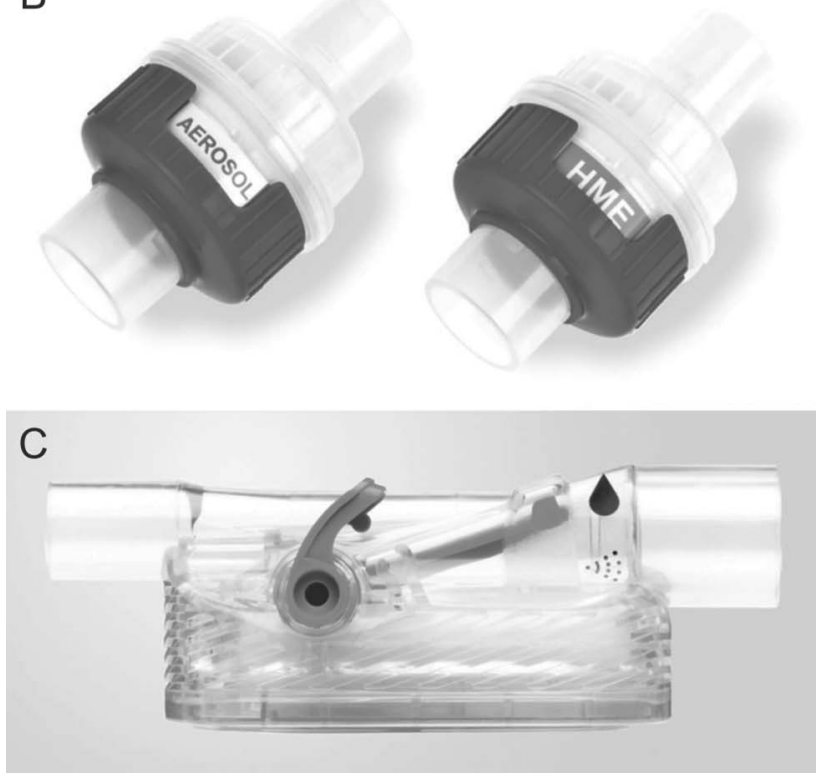

Fig. 2. Types of heat-and-moisture exchangers designed for aerosol delivery. A: CircuVent. B: Humid-Flo. C: AirLife. 


\section{Effects of HME on Aerosol Deposition in Simulated Ventilation}

\section{Data Analysis}

Drug was eluted from the collecting filter and analyzed with spectrophotometry $(276 \mathrm{~nm})$. Differences in aerosol drug delivery with and without exhaled humidity in each condition were analyzed with the dependent Student $t$ test analysis. Repeated measures of analysis of variance were used to determine differences in aerosol deposition with control and each HME-AD tested in this study. The significance level was set at .05 for data analysis $(P<.05)$.

\section{Results}

Table 3 shows the percentage of dose of albuterol delivered (mean $\pm \mathrm{SD}$ ) distal to the bronchi of the model. Without active exhaled humidity, aerosol delivery with the CircuVent, Humid-Flo, and AirLife was less than control $(P<.001, P=.01$, and $P<.001$, respectively). In this simulated ventilator-dependent lung model without exhaled humidity, the Humid-Flo had significantly higher aerosol deposition than the AirLife and the CircuVent $(P=.003$ and $P=.01$, respectively), whereas aerosol delivery with the CircuVent trended greater than the AirLife but not significant $(P=.90)$. Without exhaled humidity, a greater

Table 1. Weight, Dead Space, and Moisture Output of Heat-andMoisture Exchangers Designed to Allow Aerosol Delivery

\begin{tabular}{lccc}
\hline \hline \multicolumn{1}{c}{ HME-ADs } & Weight, g & $\begin{array}{c}\text { Dead space, } \\
\mathrm{mL}\end{array}$ & $\begin{array}{c}\text { Moisture output } \\
\left(\mathrm{V}_{\mathrm{T}}=500 \mathrm{~mL}\right), \mathrm{mg} / \mathrm{L}\end{array}$ \\
\hline Humid-Flo & 43 & 57 & 30.7 \\
AirLife & 43 & 68 & 33.5 \\
CircuVent (without an & 29.5 & 72 & Not available \\
$\quad$ HME attached to \\
the Circuvent)
\end{tabular}

amount of aerosol deposition and variation between control and the 3 HME-ADs was observed. In contrast, with exhaled humidity, no difference was found between control and any of the HME-ADs $(P=.89)$. Drug delivery without exhaled humidity exceeded aerosol deposition obtained with exhaled humidity in all conditions tested in this study.

\section{Discussion}

HME-ADs provide the opportunity to administer aerosol delivery without removing the HME from between the aerosol generator and patient airway, not breaking the circuit and maintaining positive airway pressure during aerosol therapy in mechanically ventilated patients. This is the first study that has evaluated aerosol delivery through 3 different commercially available HME-ADs using a ventilator-dependent adult lung model with and without active exhaled humidity. Results of this study showed variation between the 2 models.

With the classic model using nonhumidified exhaled gas, aerosol delivery was greater with the control than all of the HME-ADs, with differences between the 3 devices, with Humid-Flo more efficient than the CircuVent and the AirLife. In contrast, aerosol delivery with the model simulating active exhaled heat and humidity was lower, with

Table 3. Percentage of Dose Delivered Distal to the Bronchi With and Without Exhaled Humidity Using Control and Each Heat-and-Moisture Exchanger Designed to Allow Aerosol Delivery

\begin{tabular}{lcrrr}
\hline \hline \multirow{2}{*}{$\begin{array}{c}\text { Exhaled } \\
\text { Humidity }\end{array}$} & \multicolumn{4}{c}{ Dose Delivered (\%) } \\
\cline { 2 - 5 } & Control & \multicolumn{1}{c}{ AirLife } & \multicolumn{1}{c}{ CircuVent } & Humid-Flo \\
\hline With & $10.8 \pm 0.2$ & $9.2 \pm 0.7$ & $9.8 \pm 1.9$ & $9.9 \pm 1.1$ \\
Without & $18.0 \pm 0.7$ & $12.0 \pm 0.5$ & $12.6 \pm 0.8$ & $15.3 \pm 0.8$ \\
& & & & \\
\hline Results are mean \pm SD. & & & \\
\end{tabular}

Table 2. Airway Resistance Measurements With the AirLife, CircuVent, and Humid-Flo, Using a Simulated Ventilator-Dependent Adult Lung Model With and Without Exhaled Humidity

\begin{tabular}{|c|c|c|c|c|c|c|}
\hline & \multicolumn{6}{|c|}{ Airway Resistance $\left(\mathrm{cm} \mathrm{H}_{2} \mathrm{O} / \mathrm{L} / \mathrm{s}\right)$} \\
\hline & \multicolumn{3}{|c|}{ Without Exhaled Humidity } & \multicolumn{3}{|c|}{ With Exhaled Humidity } \\
\hline & AirLife & CircuVent & Humid-Flo & AirLife & CircuVent & Humid-Flo \\
\hline Before HME placement & $8.7 \pm 0.3$ & $9.7 \pm 0.3$ & $8.7 \pm 0.2$ & $10 \pm 0.1$ & $9.5 \pm 0.1$ & $10.1 \pm 0.5$ \\
\hline 5 min after HME placement & $8.8 \pm 0.2$ & $9.7 \pm 0.2$ & $8.7 \pm 0.1$ & $10.5 \pm 0.3$ & $9.6 \pm 0.2$ & $10.2 \pm 0.4$ \\
\hline 10 min after HME placement & $8.9 \pm 0.1$ & $9.8 \pm 0.2$ & $8.8 \pm 0.2$ & $11 \pm 0.4$ & $9.8 \pm 0.1$ & $10.3 \pm 0.3$ \\
\hline 15 min after HME placement & $8.9 \pm 0.2$ & $9.8 \pm 0.3$ & $8.8 \pm 0.1$ & $11 \pm 0.3$ & $9.8 \pm 0.2$ & $10.3 \pm 0.1$ \\
\hline 20 min after HME placement & $8.9 \pm 0.1$ & $9.8 \pm 0.4$ & $8.8 \pm 0.1$ & $11.0 \pm 0.2$ & $9.8 \pm 0.1$ & $10.3 \pm 0.5$ \\
\hline After treatment & $9.0 \pm 0.1$ & $10.0 \pm 0.1$ & $9.0 \pm 0.4$ & $11.3 \pm 0.5$ & $10.3 \pm 0.5$ & $10.6 \pm 0.6$ \\
\hline
\end{tabular}




\section{Effects of HME on Aerosol Deposition in Simulated Ventilation}

no difference between the control and the HME-ADs. These differences between models with passive and active humidification have substantial implications for the relevance of in vitro characterization of aerosol delivery devices.

Aerosol delivery without exhaled humidity was greater than with exhaled humidity with a greater difference between control and the HME-ADs tested in this study. We initially simulated heated humidified exhaled gas with placement of an active humidifier between the collection filter and the test lung to simulate the changes in airway resistance that occurs in HMEs over time with ventilator-dependent patients who exhale warm gas with high absolute humidity.

The effect of heated humidity versus ambient conditions during delivery of mechanical ventilation has long been associated with differences in efficiency of aerosol delivery distal to the ETT. In our previous study, ${ }^{11}$ we found $30 \%$ deposition with the dry ventilator circuit, and aerosol delivery with the heated humidified ventilator circuit was $17 \%$, which is similar to the findings of this study with the control. The difference in aerosol deposition between the dry and wet ventilator circuit using the classic model may be due in part to the lack of exhaled humidity in the lung model used in our previous research. Using a model with exhaled humidity reduced aerosol deposition distal to the ETT to levels previously associated with use of heated humidification.

Active exhaled humidity in the in vitro model reduces aerosol drug delivery compared with passive unheated exhalation. In this model, as with patients, exhaled gas has a high absolute humidity. As dry air is delivered by the ventilator, it is heated and humidified in transit through the airway, where growth of particles has been described as almost instantaneous, increasing impactive losses. ${ }^{12}$ With unheated passive exhalation aerosol particles in dry air do not reach the high absolute humidity required for particle growth during transit through the airway.

Lin et $\mathrm{al}^{13}$ reported that during the first hour after turning on a heated humidifier during mechanical ventilation, aerosol delivery via pressurized metered-dose inhaler was similar to the dry ventilator circuit. Subsequent aerosol delivery in the heated circuit was decreased at a level described previously. However, turning off the heated humidifier for up to $20 \mathrm{~min}$ before aerosol administration did not improve delivery efficiency of the pressurized metered-dose inhaler back to the level of a dry circuit. The authors suggested that the formation and presence of condensate increase absolute humidity in the circuit, which leads to a reduction in aerosol delivery to ventilated patients. Since we observed considerable condensate in the airway and ventilator circuit between the HME and the collecting filter, we believe that condensate may have a similar effect on humidification, and previously reported reduction in aerosol deposition between heated and dry circuits may be in part due to an artifact of in vitro lung models that do not simulate exhaled humidity.

Placement of collecting filters distal to the bifurcation of an anatomic model provides a more representative airway surface and volume than filter placement immediately distal to the endotracheal tube. In additional, prior experiments of collecting filters in both heated humidified and non-heated conditions established similar filter efficiency of medical aerosols.

Further research is warranted to better understand the effect of exhaled heated and humidified gas through artificial airways, with resulting condensate formation, and to determine the magnitude of deposition efficiency in patients receiving aerosol with heated and humidified versus dry gas.

\section{Conclusions}

In this model simulating active exhaled humidity, aerosol drug delivery was lower and more consistent with both control and the HME-ADs than with the standard nonhumidified adult model of mechanical ventilation. Further studies are needed to determine whether greater deposition in a dry model is an artifact of a model that does not simulate active exhaled humidity in patients.

\section{REFERENCES}

1. American Association for Respiratory Care, Restrepo RD, Walsh BK. Humidification during invasive and noninvasive mechanical ventilation: 2012. Respir Care 2012;57(5):782-788.

2. International Organization for Standardization. Heat and moisture exchangers for use in humidifying respired gases in humans (ISO 9360). Geneva: International Organization for Standardization; 1992.

3. Respiratory tract humidifiers for medical use-particular requirements for respiratory humidification systems. ISO 8185: 2007. https:// www.iso.org/obp/ui/\#iso:std:iso:8185:ed-3:v2:en. Accessed November 1, 2016.

4. Thomachot L, Leone M, Razzouk K, Antonini F, Vialet R, Martin C. Randomized clinical trial of extended use of a hydrophobic condenser humidifier: 1 vs. 7 days. Crit Care Med 2002;30(1):232-237.

5. Davis K Jr, Evans SL, Campbell RS, Johannigman JA, Luchette FA, Porembka DT, Branson RD. Prolonged use of heat and moisture exchangers does not affect device efficiency or frequency rate of nosocomial pneumonia. Crit Care Med 2000;28(5):1412-1418.

6. Chiaranda M, Verona L, Pinamonti O, Dominioni L, Minoja G, Conti G. Use of heat and moisture exchanging (HME) filters in mechanically ventilated ICU patients: influence on airway flow-resistance. Intensive Care Med 19(8)93;19:462-466.

7. Kubiak BD, Albert SP, Gatto LA, Trikha G, El-Zammar O, Nieman GF. Loss of airway pressure during HFOV results in an extended loss of oxygenation: a retrospective animal study. J Surg Res 2010; 162(2):250-257.

8. American Association for Respiratory Care. AARC clinical practice guidelines: endotracheal suctioning of mechanically ventilated patients with artificial airways. Respir Care. 2010;55(6):758-764.

9. Craven DE, Goularte TA, Make BJ. Contaminated condensate in mechanical ventilator circuits: a risk factor for nosocomial pneumonia. Am Rev Respir Dis 1984;129(4):625-628. 


\section{Effects of HME on Aerosol Deposition in Simulated Ventilation}

10. Craven D, Kunches LM, Kilinsky V, Lichtenberg DA, Make BJ, McCabe WR. Risk factors for pneumonia and fatality in patients receiving continuous mechanical ventilation. Am Rev Respir Dis 1986;133(5):792-796.

11. Ari A, Areabi H, Fink JB. Evaluation of position of aerosol device in two different ventilator circuits during mechanical ventilation. Respir Care 2010;55(7):837-844.
12. Lange CF, Finlay WH. Overcoming the adverse effect of humidity in aerosol delivery via pressurized metered-dose inhalers during mechanical ventilation. Am J Respir Crit Care Med 2000;161(5):16141618.

13. Lin HL, Fink JB, Zhou Y, Cheng YS. Influence of moisture accumulation in inline spacer on delivery of aerosol using metered-dose inhaler during mechanical ventilation. Respir Care 2009;54(10):1336-1341.

This article is approved for Continuing Respiratory Care Education credit. For information and to obtain your CRCE

(free to AARC members) visit 